\title{
A study on the potential of carbon residue from rice husk used as boiler fuel for carbon dioxide capture and wastewater treatment
}

\author{
Janssen Radley Peñaflor ${ }^{1}$, Airic James Carillo ${ }^{1}$, Samuel Elijah Estrada ${ }^{1}$, Jhulimar Celedonio-Castro ${ }^{1,2^{*}}$ \\ ${ }^{1}$ Department of Chemical Engineering, University of Santo Tomas, España, Manila 1008, Philippines \\ ${ }^{2}$ Research Center for Natural and Applied Sciences, University of Santo Tomas, España, Manila 1008, Philippines
}

\begin{abstract}
Adsorption process both in post carbon dioxide capture and wastewater treatment has been receiving widespread attention over the past decades as a mitigating technology for climate change and water pollution, respectively. With this increasing interest in adsorption processes to address environmental concerns, development of an adsorbent with not just high adsorptive capacity but which is also low cost is of great interest among researchers. In this study, an agricultural waste which was already utilized as a boiler fuel in a paper industry was investigated for its potential as an adsorbent for both carbon dioxide capture and wastewater treatment. Specifically, the $\mathrm{CO} 2$ and methylene blue adsorption capacity of carbon residue from rice husk used as boiler fuel was determined and was compared with a biochar synthesized from rice husk. Furthermore, Scanning Electron Microscope (SEM) and Thermogravimetric analysis (TGA) were used for the characterization of the adsorbents. Results showed that the carbon residue can be a potential adsorbent for both applications with about $0.5 \mathrm{wt} \% \mathrm{CO} 2$ adsorption and $100 \%$ removal of the methylene blue.
\end{abstract}

\section{Introduction}

Philippines is known to be mainly an agricultural country. The country's total land area of 298,170 square kilometers is known to be 47 percent agricultural lands. This agricultural land area is devoted to several crops and food grains. Among the several crops grown, the main contributors include rice, coconut, corn, banana and sugarcane, wherein rice is the highest contributor. For the years 2011 to 2014, the average volume of rice production is $18,054,720$ metric tons [1].

Behind the high production volumes of these crops, its by-products are also considered as the highest contributors of agricultural wastes [2]. Agricultural wastes are non-consumable by-products of the production of agricultural products wherein other processes including its transportation, storage and post-processes are costlier than its value. Due to the large amount of abandoned agricultural wastes, several agro-industrial wastes utilization applications are being developed. These technologies include fertilizer application, anaerobic digestion, ethanol production, energy production from biomass, pyrolysis, direct combustion and as an adsorbent in wastewater treatment and carbon dioxide capture $[3,4]$.

One way to utilize agro-industrial wastes is by using it as an adsorbent. This separation process is known as the accumulation of a substance at the interface between two different phases namely adsorbent and adsorbate [5]. Adsorption has been proven by several researches as a reliable process in treating several industrial wastes including pollutants from water such as dye and heavy metals and air pollutants, for instance, carbon dioxide.

With the several agro-industrial wastes present, rice husk may be highly utilized due to the large volumes of production of rice. One of the applications of rice husk is to function as an adsorbent in the removal of antimony, cadmium, lead, dyes and phenol in aqueous solutions [6]. Another application is in $\mathrm{CO}_{2}$ capture where activated carbon is considered as a well-known adsorbent. Besides the mesoporous carbonated structures, they also have a surface chemistry which can be modified by different methodologies [7].

The main objective of the study is to investigate on the potential use of agro-industrial waste, specifically carbon residue derived from rice husk used as boiler fuel in a paper industry, in capturing carbon dioxide and treating wastewater. Specifically, the study aims:

(1) To determine the $\mathrm{CO}_{2}$ adsorption capacity and thermal stability of the carbon residue;

(2) To determine the adsorption capacity of the adsorbent in treating a methylene blue solution;

(3) To compare the $\mathrm{CO}_{2}$ and methylene blue adsorption capacity of the carbon residue to another form of carbon derived from rice husk by pyrolysis, biochar.

\footnotetext{
* Corresponding author: jmceledonio@ust.edu.ph
} 


\section{Materials and methods}

\subsection{Materials and reagents}

The carbon residue was obtained from Sterling Paper Products Ent. Inc. Plant in Marilao, Bulacan. The carbon residue was derived from rice husk which serves as a fuel for the plant's boiler. At isolated conditions, the rice husk was burned at a temperature of $800^{\circ} \mathrm{C}$. On the other hand, rice husk was obtained from Cabanatuan, Nueva Ecija and was pyrolized at $600^{\circ} \mathrm{C}$ to produce biochar.

High purity nitrogen gas and $\mathrm{CO}_{2}$ gas (Atgas Traders) were used for the determination of the thermal stability and $\mathrm{CO}_{2}$ adsorption capacity of the carbon materials. For the simulation of wastewater treatment, methylene blue was used to prepare a colored solution. Ultrapure water was used in all solution preparations, washing and rinsing of glass wares.

\subsection{Experimental set-up}

\subsubsection{Preparation of carbon residue and biochar}

For a smaller and more uniform particle size of the adsorbent, the carbon residue was sieved using the RX29-16 Sieve Shaker with a sieve tray of 150 mesh. Meanwhile, the rice husk was milled using the Willey Mill to reduce its size and then sieved using the Sieve Shaker with a sieve tray of 150 mesh. After which, the sieved rice husk was pyrolized at $600{ }^{\circ} \mathrm{C}$ using a muffle furnace.

Both the carbon residue and biochar were then washed and filtered for 5 times using ultrapure water with a ratio of $100 \mathrm{~mL}$ ultrapure water to $10 \mathrm{~g}$ of carbon materials. Thereafter, the filtered carbon materials were oven-dried at $105^{\circ} \mathrm{C}$

\subsubsection{Characterization of adsorbents}

The carbon materials were subjected to Scanning Electron Microscope (SEM) analysis using a TM3000 Tabletop Microscope (Hitachi) under vacuum conditions. The analysis was done to determine the surface morphology of the samples. The thermal stabilities of the carbon materials under pure nitrogen gas and air from $30^{\circ} \mathrm{C}$ to $300^{\circ} \mathrm{C}$ ramped at a rate of $5{ }^{\circ} \mathrm{C} / \mathrm{min}$ were analyzed using a Perkin-Elmer Thermogravimetric Analyzer.

\subsubsection{Evaluation of applicability to carbon dioxide capture}

The $\mathrm{CO}_{2}$ adsorption capacity of the adsorbents were analyzed using a Perkin-Elmer Thermogravimetric Analyzer. Pre-adsorbed moisture and $\mathrm{CO}_{2}$ from the atmosphere were purged at $150^{\circ} \mathrm{C}$ under nitrogen gas flowing at $20 \mathrm{~mL} / \mathrm{min}$ prior to any measurement. The $\mathrm{CO}_{2}$ adsorption capacity of the carbon materials was measured isothermally at $30^{\circ} \mathrm{C}$ by exposing the carbon materials under flowing carbon dioxide gas at a flowrate of 50 $\mathrm{mL} / \mathrm{min}$ for 60 minutes.

\subsubsection{Evaluation of applicability to wastewater Treatment}

The wastewater treatment experiment was done using methylene blue as the adsorbate. The percent removal of the dye in $150 \mathrm{~mL}$ of $50 \mathrm{ppm}, 75 \mathrm{ppm}$ and $100 \mathrm{ppm}$ methylene blue solutions were measured using $0.75 \mathrm{~g}$ of adsorbents. The removal of dye was also measured as a function of time by monitoring the percent removal every 10 minutes for a span of 90 minutes.

\section{Results and discussion}

\subsection{Characterization of adsorbents}

The SEM images show the surface transformation of rice husk after pyrolysis and burning. Fig. 1 shows the surface of the sieved rice husk while Figs. $\mathbf{2 a}$ and $\mathbf{2 b}$ show the surface of the rice husk after pyrolysis and burning, respectively.

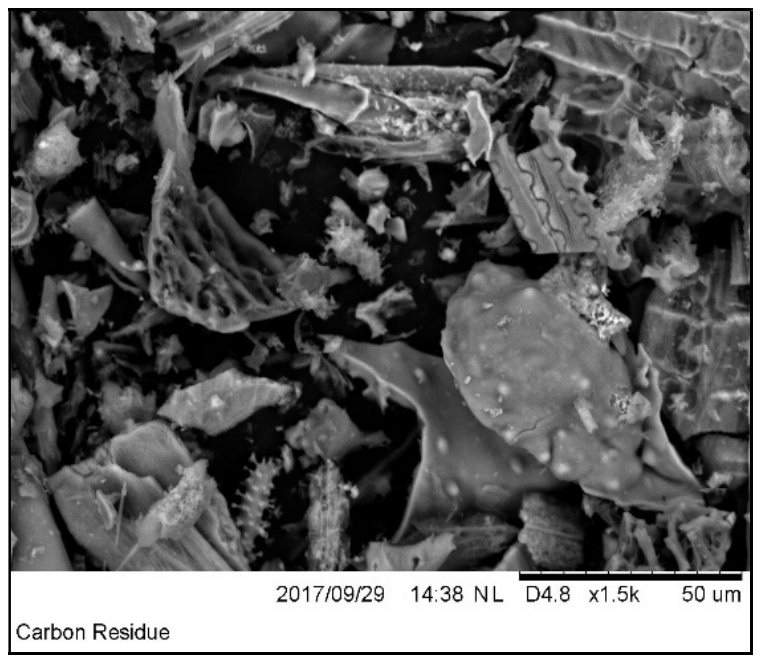

Figure 1. SEM image of rice husk at x1500 magnification.

SEM image of the rice husk in Fig. 1 shows smooth surface with little to neither flaky layers nor pores. The absence of pores indicates unavailability of adsorption sites. However, after pyrolysis and burning, as shown in Fig. 2(a) and Fig. 2(b), respectively, adsorption sites in the form of flaky layers and pores have been observed. 


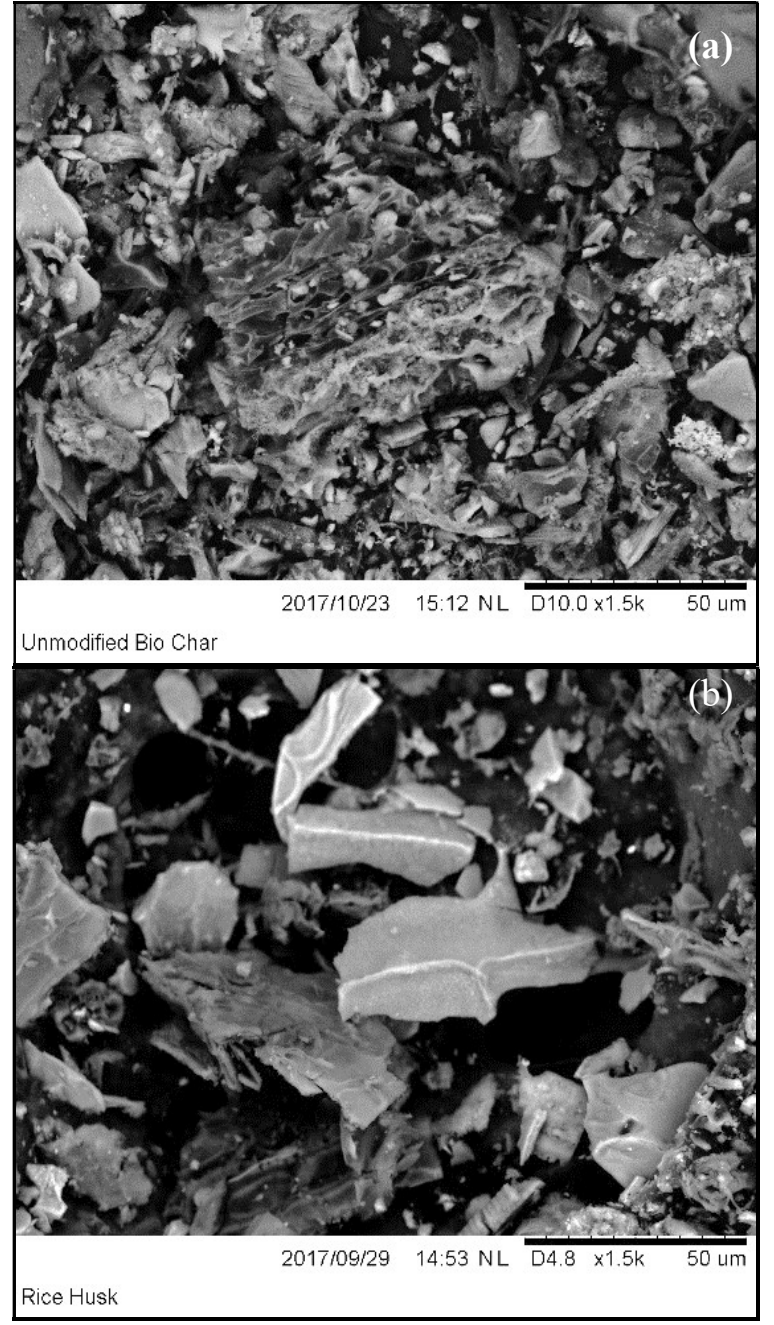

Figure 2. SEM image of (a) biochar and (b) carbon residue at x1500 magnification.

\subsection{Evaluation of applicability to carbon dioxide capture}

To determine the thermal stability of the carbon materials for carbon capture application, the adsorbents were exposed to different gases from $30{ }^{\circ} \mathrm{C}$ to $300{ }^{\circ} \mathrm{C}$ at 5 ${ }^{\circ} \mathrm{C} /$ min ramping rate using thermogravimetric analyzer. Fig. 3 and Fig. 4 show the thermal analysis under nitrogen gas and ambient air, respectively. From these figures, it can be seen that the adsorbents initially adsorbed about $0.2-0.4 \mathrm{wt} \%$ of the gas due to the sudden increase in flowrate from $20 \mathrm{ml} / \mathrm{min}$ during pre-treatment to $50 \mathrm{ml} / \mathrm{min}$ during analysis. However, the adsorbed gas was eventually released as the temperature increases. The further decreased in mass as the temperate increases can be attributed to pre-adsorbed humidity, carbon dioxide and other adsorbed substances that were not totally removed during pre-treatment. This observation suggests that that the carbon materials are not suitable for hightemperature adsorption since it desorbs gases at high temperature. However, this is favorable for carbon sequestration and proves the reusability of the adsorbent. Through heating of the used adsorbent, the carbon dioxide captured may be displaced to a safer environment or may be used in other processes. Moreover, the carbon materials are proved to be thermally stable since it has not been decomposed at higher temperature.

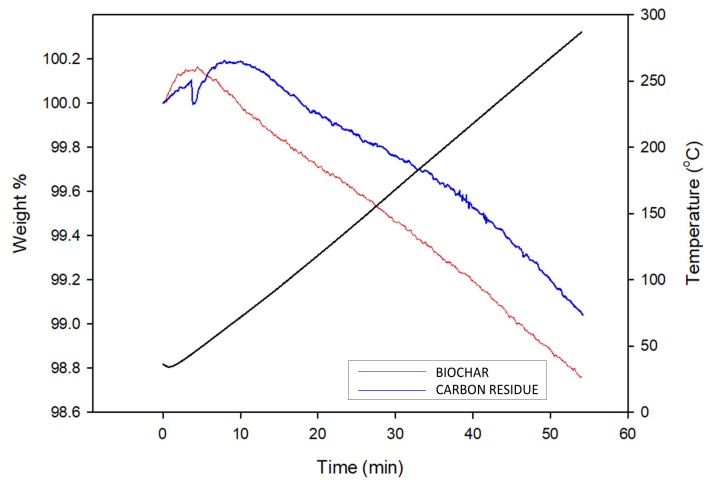

Figure 3. Thermal stability of the carbon materials under nitrogen gas.

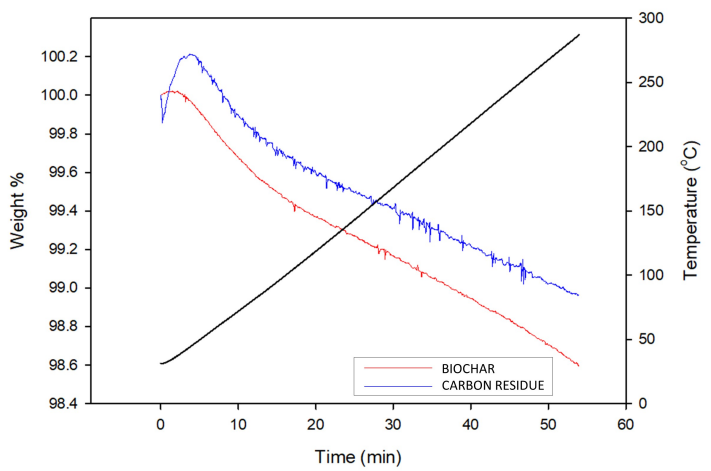

Figure 4. Thermal stability of the carbon materials under ambient air.

After which, the capability of the carbon materials to capture $\mathrm{CO}_{2}$ at ambient condition was determined by exposing the carbon materials to flowing carbon dioxide gas at $30^{\circ} \mathrm{C}$ at a flowrate of $50 \mathrm{~mL} / \mathrm{min}$ for 60 minutes. Fig. 5 shows this adsorption of carbon dioxide by the samples as can be seen in the change in the weight immediately upon exposure to carbon dioxide. The higher $\mathrm{CO}_{2}$ adsorption capacity of $1.1 \mathrm{wt} \%$ of the biochar compared to the $0.5 \mathrm{wt} \% \mathrm{CO}_{2}$ adsorption capacity of the carbon residue can be attributed to the difference of their activation temperature that could have caused difference in surface morphology. Based from the study of Shoaib, et. al, higher activation temperature causes smaller pore size but bigger surface area and pore volume [8]. Following this, carbon residue burned at $800^{\circ} \mathrm{C}$ will have smaller pore size and higher surface area compared to biochar pyrolyzed at $600^{\circ} \mathrm{C}$. Therefore, the possibility of carbon dioxide adsorbed on the surface blocking the pores' inner surfaces caused the lower adsorption of carbon residue than biochar. 


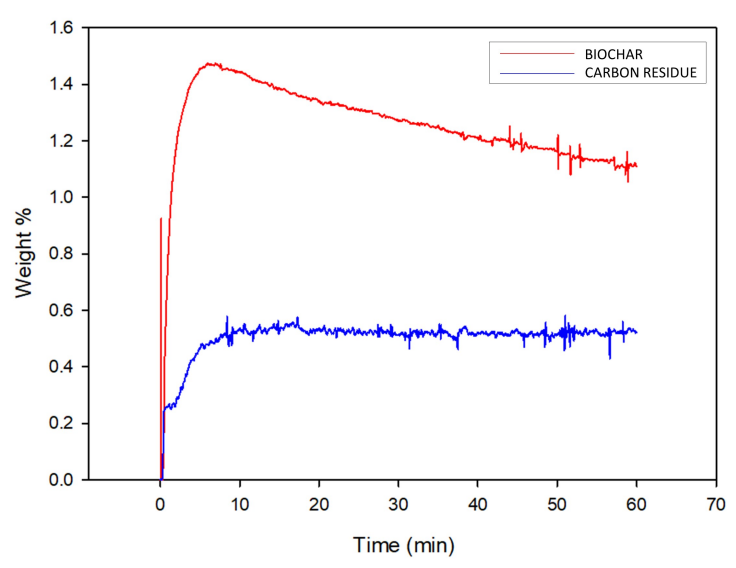

Figure 5. Single Sorption Capacity of Unmodified and AmineModified Carbon Residue

\subsection{Evaluation of applicability to wastewater treatment}

To determine the applicability of the carbon materials for wastewater treatment, the percent removal of dye in simulated wastewater from paper and textile industries, specifically methylene blue solutions with concentrations of $50 \mathrm{ppm}, 75 \mathrm{ppm}$ and $100 \mathrm{ppm}$, was measured as a function of time using $0.75 \mathrm{~g}$ of the carbon materials.

Fig. 6 shows the percent removal of methylene blue in $75 \mathrm{ppm}$ solution using $0.75 \mathrm{~g}$ of the carbon materials. As can be seen on the figure, the adsorbents were capable of adsorbing methylene blue and were able to remove up to $97 \%$ of the dye using carbon residue and $44 \%$ using biochar. The removal slowly increases with time which may be attributed to the kinetics of adsorption. Spikes at 60 minutes of adsorption may be attributed to errors.

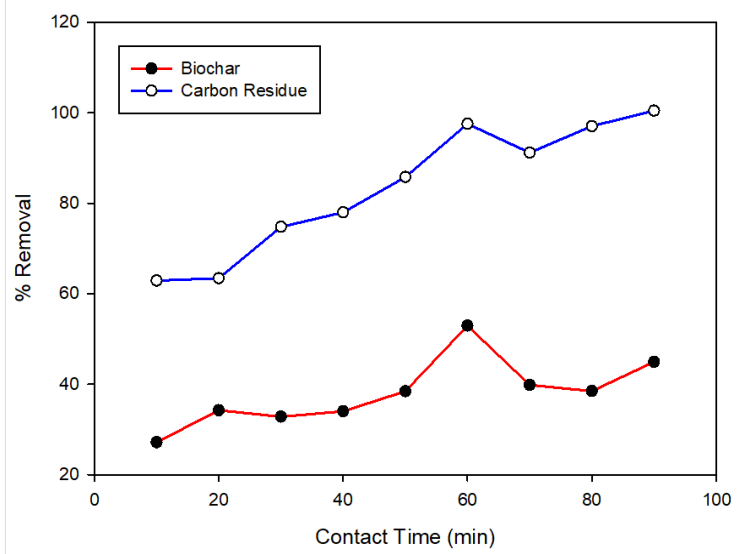

Figure 6. Effect of Time on the \% Removal of Carbon Residue and Biochar

Meanwhile, Fig. 7 shows the percent removal of methylene blue in $50 \mathrm{ppm}, 75 \mathrm{ppm}$ and $100 \mathrm{ppm}$ of solution using $0.75 \mathrm{~g}$ of the carbon materials.

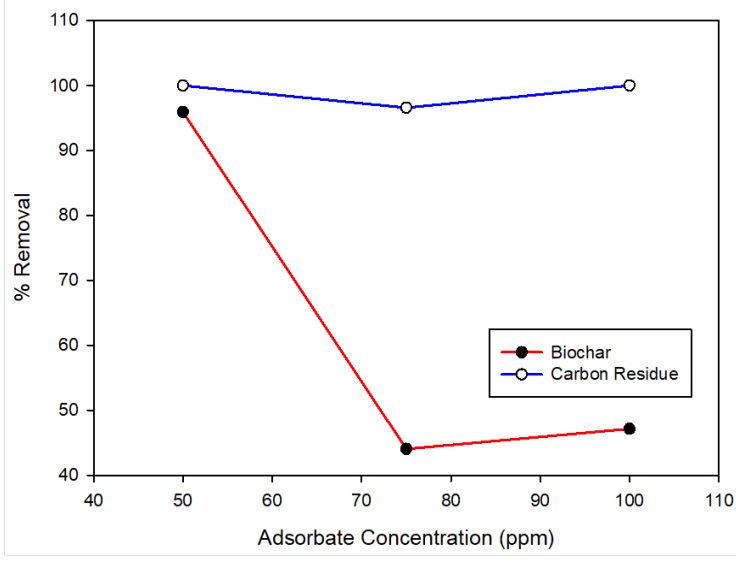

Figure 7. \% Removal of the Adsorbents in Varying Adsorbate Concentration Test

The data shows that both biochar and carbon residue was able to achieve high removal of the methylene blue, at $96 \%$ and $100 \%$, respectively, when the concentration was at $50 \mathrm{ppm}$. The carbon residue showed great aptitude for methylene blue removal even at high methylene blue concentrations. In contrast, biochar showed decreasing capability for adsorption at higher concentration of methylene blue. It shows that biochar is only suited for the adsorption of less concentrated methylene blue. This trend can be attributed to the predicted smaller surface area of biochar compared to carbon residue.

\section{Conclusion}

The research was able to show that rice husk, an agroindustrial waste, in the form of carbon residue after it has been used as boiler fuel is capable to be utilized as an adsorbent in both carbon dioxide capture and wastewater treatment application. Furthermore, the carbon residue was compared to biochar which is another form of carbon material derived from rice husk by pyrolysis.

Both biochar and carbon residue showed opening of pores compared to rice husk under SEM. In terms of thermal stability for $\mathrm{CO}_{2}$ capture application, both carbon materials were found to be thermally stable under nitrogen and ambient air. Both materials did not show degradation upon exposure to both gases up to $300^{\circ} \mathrm{C}$. In terms of $\mathrm{CO}_{2}$ adsorption capacity, carbon residue showed lower capacity of $0.5 \mathrm{wt} \%$ compared to that of biochar of $1.1 \mathrm{wt} \%$. In terms of methylene blue adsorption, carbon residue showed higher removal of methylene blue compared to biochar, removing $97 \%$ and $44 \%$ methylene blue in $75 \mathrm{ppm}$ solutions, respectively. However, in $50 \mathrm{ppm}$ solution, both carbon residue and biochar showed high removal at $100 \%$ and $96 \%$, respectively. Nevertheless, in all 50, 75 and 100 ppm solutions, carbon residue showed better adsorption than biochar.

The difference in the trend for carbon capture and wastewater treatment can be attributed to the possible difference in surface area and pore volume of the two carbon materials brought by the two-different pore activation processes they undergone. 
This research was financially supported by the Research Center for the Natural and Applied Sciences of the University of Santo Tomas (UST RCNAS).

\section{References}

[1] Philippine Statistics Authority, 2014.

[2] Baconguis, S. R. Abandoned Biomass Resource Statistics in the Philippines. 10th National Convention on Statistics, 2007.

[3] Obi, F. O., Ugwuishiwu, B. O., \& Nwakaire, J. N. Agricultural Waste Concept, Generation, Utilization and Management. Nigerian Journal of Technology 35(4), 2016, pp. 957-964.

[4] Zhang, C., Song, W., Sun, G., Xie, L., Wang, J., $\mathrm{Li}, \mathrm{K}$. , Drage, T. $\mathrm{CO}_{2}$ capture with activated carbon grafted by nitrogenous functional groups. Energy and Fuels 27(8), 2013, pp. 4818-4823.

[5] Fu, L., Zhang, G., Wang, S., Zhang, L., \& Peng, J. Modification of activated carbon via grafting polyethyleneimine to remove amaranth from water. Applied Water Science 7(8), 2017, pp. 4247-4254.

[6] Bhatnagar, A., \& Sillanpää, M. Utilization of agro-industrial and municipal waste materials as potential adsorbents for water treatment-A review. Chemical Engineering Journal 157(2-3), 2010, pp. 277-296.

[7] Wang, P., Guo, Y., Zhao, C., Yan, J., \& Lu, P. Biomass derived wood ash with amine modification for post-combustion $\mathrm{CO}_{2}$ capture. Applied Energy 201, 2017, pp. 34-44.

[8] Shoaib, M., \& Al-swaidan, H. M. Optimization of activation temperature on the preparation of sliced porous activated carbon from date fronds by physical activation. Hemijska industrija (Chemical Industry) 70 (2), 2016, pp. 151-157 